Pacific Journal of Mathematics

REPRESENTATIONS OF BRAID GROUPS AND THE QUANTUM
YANG-BAXTER EQUATION 


\title{
REPRESENTATIONS OF BRAID GROUPS AND THE QUANTUM YANG-BAXTER EQUATION
}

\section{HANS WENZL}

\begin{abstract}
We are going to study the construction of new representations of braid groups and solutions of quantum Yang-Baxter (=QYBE) from existing ones via cabling. This can be applied for the construction of new link invariants from a given one for a wide class of invariants. For the example of the 2-variable generalization of the Jones polynomial, this yields for each Young diagram a 1-parameter family of representations of the braid groups and a 2-variable link invariant. Using the braid representations from the QYBE, one obtains a 1-variable link invariant for each irreducible representation of a classical Lie algebra.
\end{abstract}

It is well known that one obtains an injective homomorphism from $B_{\infty}$ (which can be thought of as the inductive limit of the finite braid groups) into itself by cabling, i.e. by replacing each string of a braid $\beta \in B_{\infty}$ by $f$ parallel strings (see figures in $\S 1$ ). Composing this homomorphism with a given representation $\rho$ of $B_{\infty}$ yields a new representation of $\rho^{(f)}$ of $B_{\infty}$. Similarly as with higher tensor product representations, one can decompose the cabled representation of $B_{\infty}$. If $\rho\left(\mathbf{C} B_{f}\right)$ is finite dimensional, the subrepresentations are given in terms of minimal idempotents of $\rho\left(\mathbf{C} B_{f}\right)$. More representations can be constructed by applying additional twists on the cabled braids. We will show that while the full representations may be different special subrepresentations are the same for all these twistings.

In the approach by Jones, link invariants were constructed by finding special traces on $\mathbf{C} B_{\infty}$, the so-called Markov traces. On the other hand, each link invariant with values in a field $k$ and a minor additional condition can be used to define a Markov trace on $k B_{\infty}$. Moreover, there is a well-known construction (known to operator algebraists as GNS construction) by which one obtains a representation of $B_{\infty}$ from a Markov trace. We will apply this correspondence between link invariants, Markov traces and representations of braid groups to construct new link invariants from existing ones via cabling and decomposition of the corresponding braid representations. Essentially the same observation has been made by J. Murakami in his approach of computing cabled line invariants (see $[\mathbf{M}])$. In this section, 
we will use his approach to prove the Markov property of the newly constructed traces. Applying this to the example of the [FYHLMO] 2-variable polynomial, we obtain for each Young diagram a 2-variable link invariant. If $\lambda^{*}$ is the Young diagram obtained from $\lambda$ by interchanging rows with columns, the corresponding link invariant of $\lambda^{*}$ can be obtained from the one of $\lambda$ by a simple substitution of variables. On the other hand we show that the representations belonging to Young diagrams $[n]$, i.e. diagrams with $n$ boxes in only one row, are not equivalent for different $n$. It follows from computations by Murakami, Morton-Short and Yamada that these new invariants can distinguish links which are not distinguished by the original one.

In the next section we present a different method of proof for the existence of cabled link invariants which has some similarities with Weyl's unitarian trick. As the link invariants are holomorphic functions, it suffices to check this for special values of the parameters. These parameters are chosen such that one obtains a unitary representation of the braid groups. In this case, results about the corresponding von Neumann algebras can be applied to show that we have a link invariant at these special values. The general statement then follows from the principle of isolated zeros. With this method one can also prove additional properties of these link invariants such as multiplicativity for products of knots.

The cabling approach can also be used to construct new explicit solutions of the quantum Yang-Baxter equations (QYBE) from existing ones. Solutions of these equations have been obtained by Drinfeld and Jimbo as commuting objects of the second tensor products of deformations of Lie algebras (so-called quantum groups) in their standard representations. The solutions coming from the $f$-cabling can be naturally understood as commuting objects of the quantum groups in the $f$ th tensor product of their standard representations. Setting the 'spectral parameter' of the solutions of QYBE equal to 0 , one obtains representations of the braid groups.

For Lie type $A$ it has been shown by Jimbo that these representations map into quotients of the Hecke algebras of type $A$. This extends the classical Schur-Weyl duality to a duality between quantum groups of type $A$ and Hecke algebras of type $A$. The connection between the QYBE and Jones' work on subfactors and link invariants was realized when the author noticed that Jimbo's representations for type $A_{1}$ coincide with representations of Pimsner and Popa who also found an easy description of the Markov trace in terms of a product 
state. This can be easily generalized to type $A_{k}$. Jones subsequently succeeded in constructing the corresponding link invariants directly from quantum statistical models without using braids and traces. This was generalized by Turaev.

Applying the $f$-cabling approach to these braid representations, the newly constructed link invariants correspond naturally to irreducible representations appearing in the $f$ th tensor power of the standard representation of quantum groups of type $A$. This can also be done in general for other Lie algebras (see [W-3] for types $B, C$ and $D$ ). Together with the results of [W-3] it follows that one has a one-variable link invariant for each irreducible finite dimensional representation of a classical complex Lie algebra (with some minor modifications for type $D$ ). These results have also been obtained independently and by different methods by Akutsu and Wadati [AW] and Jones [J-2] in special cases and by Rosso [Ro] and Reshetikhin [Re] in general. This paper is an expanded version of [W-4].

1. Representations of braid groups via cabling. Let $B_{\infty}$ be the infinite braid group, given by generators $\sigma_{1}, \sigma_{2}, \ldots$ and relations

(B1) $\sigma_{1} \sigma_{i+1} \sigma_{i}=\sigma_{i+1} \sigma_{i} \sigma_{i+1}$ for $i=1,2, \ldots$ and

(B2) $\sigma_{i} \sigma_{j}=\sigma_{j} \sigma_{i}$ if $|i-j| \geq 2$.

The subgroup of $B_{\infty}$, which is generated by $\sigma_{r}, \sigma_{r+1}, \ldots, \sigma_{s-2}$, $\sigma_{s-1}$ is denoted by $B_{r, s}$ and $B_{1, f}$ will be abbreviated by $B_{f}$.

One of the usual models for $B_{n}$ is as follows (see [BZ] or [B] for details): Fix on each of 2 parallel lines in 3 space (an 'upper' and a 'lower' one) $n$ points labelled by the numbers $1,2, \ldots, n$. We also require that this labelling respects the natural ordering of our index set. A braid is obtained by connecting each of the upper points with a point of the lower line by a (smooth or piecewise linear) curve going only downwards and by taking the isotopy class of this object (with fixed end points). Multiplication of 2 braids is defined by connecting the lower points of the first braid with the upper points of the second braid with matching labels. Similarly $B_{\infty}$ can be realized by countably many points such that all but finitely many of them go straight downwards. It has been shown by $\mathrm{E}$. Artin that $\sigma_{i}$ can be realized in this model by the object in Figure 1.

We will call an element of a braid group in this model a braid projection. Let

$$
\Delta_{r, s}=\left(\sigma_{r} \sigma_{r+1} \cdots \sigma_{s-1}\right)\left(\sigma_{r} \sigma_{r+1} \cdots \sigma_{s-2}\right) \cdots \sigma_{r} .
$$

Then the center of $B_{r, s}$ is generated by $\Delta_{r, s}^{2}=\left(\sigma_{r} \sigma_{r+1} \cdots \sigma_{s-1}\right)^{(s-r+1)}$. 


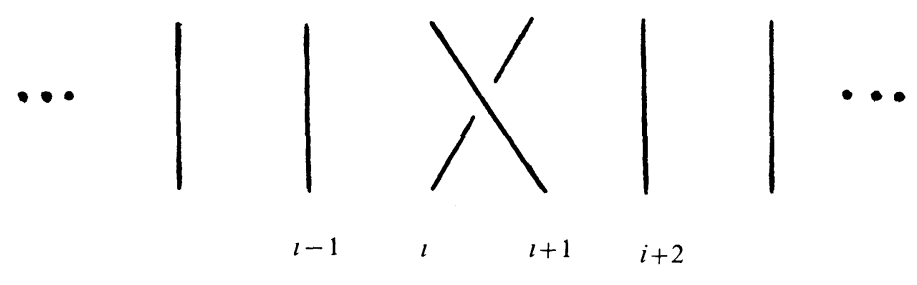

FIGURE 1

The elements $\Delta_{r, s}$ and $\Delta_{r, s}^{2}$ are called the half twist and the full twist of $B_{r, s}$ respectively. As before, we write $\Delta_{f}$ for $\Delta_{1, f}$ (see [B] for the geometric interpretation of these braids).

Let now the braid $\sigma_{i}^{(f)}$ be given by the upper part of Figure 2.

It is easy to check by pictures that $\sigma_{i} \mapsto \sigma_{i}^{(f)}$ induces an injective homomorphism from $B_{\infty}$ into itself (we only replace a single string by $f$ strings). Similar homomorphisms are also obtained with additional compatible twists. More precisely, let $\beta, \gamma \in B_{f}$ and let $\sigma_{i}^{(f, \beta, \gamma)}$ be the braid obtained by adding $\beta$ and $\gamma$ to $\sigma_{i}^{(f)}$ as indicated in Figure 2 . Then it is again easy to check by pictures that

$$
\text { The map } \sigma_{i} \mapsto \sigma_{i}^{(f, \beta, \gamma)} \text { extends to an (injective) }
$$

homomorphism of $B_{\infty}$ into $B_{\infty} \Leftrightarrow \beta$ and $\gamma$ commute.

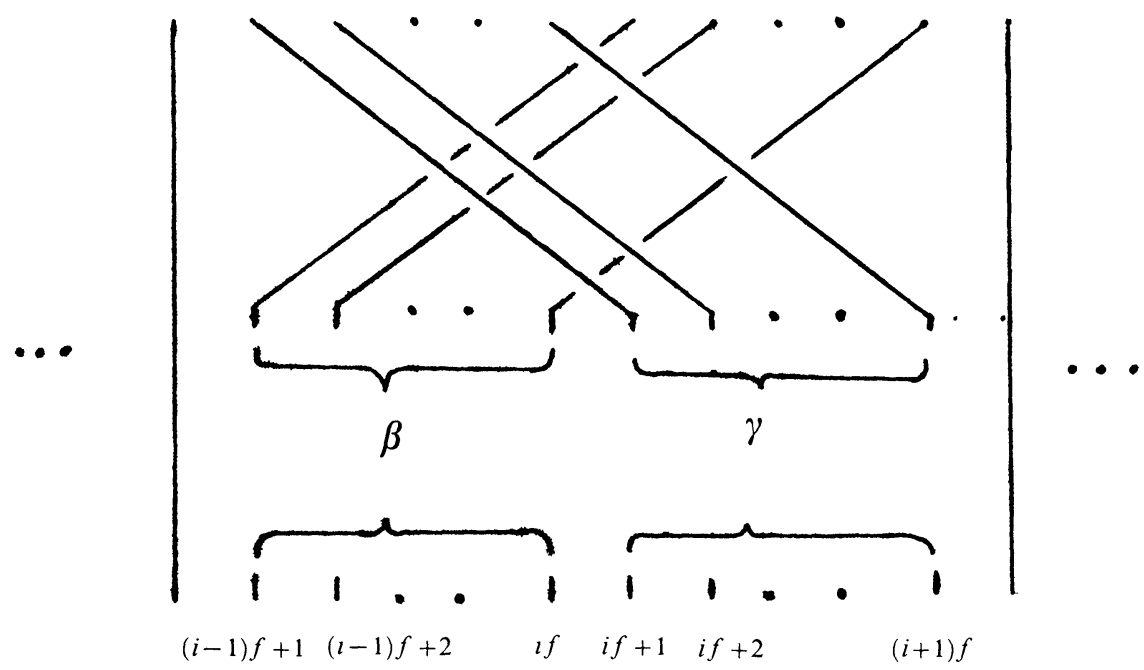

FIGURE 2 
If $\rho$ is an arbitrary representation of $B_{\infty}$, such that $\rho(\beta)$ and $\rho(\gamma)$ commute, one obtains a new representation $\rho^{(f, \beta, \gamma)}$ by

$$
\rho^{(f, \beta, \gamma)}\left(\sigma_{i}\right)=\rho\left(\sigma_{i}^{(f, \beta, \gamma)}\right) .
$$

If $\beta=\gamma=1$, we will just write $\rho^{(f)}$ for $\rho^{(f, \beta, \gamma)}$. In the following let $k$ be a field and let $\rho$ be a representation of $B_{\infty}$. We will say that $\rho$ is an AFD (= approximately finite dimensional) representation of $B_{\infty}$, if $\rho\left(k B_{n}\right)$ is finite dimensional and semisimple for all $n \in \mathbf{N}$. As we will only be interested in the cases $k=\mathbf{C}$ or $k$ a field of rational functions in one or several variables over $\mathbf{C}$, semisimple here means that $\rho\left(k B_{n}\right)$ is a direct sum of full matrix algebras.

We are now going to decompose these representations. Observe that conjugation by $\sigma_{i}^{(f)}$ interchanges $\sigma_{j}$ and $\sigma_{j+f}$ for $j=(i-1) f+1$, $(i-1) f+2, \ldots$, if -1 . This is used to define inductively for any element $\beta \in B_{f}$ new elements $\beta_{i} \in B_{\infty}$ by

$$
\beta_{1}=\beta \quad \text { and } \quad \beta_{i+1}=\sigma_{i}^{(f)} \beta_{i}\left(\sigma_{i}^{(f)}\right)^{-1} .
$$

The element $\beta_{i+1}$ can be thought of as $\beta_{i}$ being 'shifted' to the right by $f$ strings. Observe that we also have

$$
\beta_{i}=\sigma_{i}^{(f)} \beta_{i+1}\left(\sigma_{i}^{(f)}\right)^{-1} \text {. }
$$

So conjugation by $\sigma_{i}^{(f)}$ permutes $\beta_{i}$ and $\beta_{i+1}$. Furthermore let $\beta_{(n)}=\beta_{1} \beta_{2} \cdots \beta_{n}$. Similarly, if $\rho$ is a representation of $B_{\infty}$, we define for elements $b \in \rho\left(k B_{f}\right)$ elements $b_{i}$ and $b_{(n)}$ by conjugation by $\rho\left(\sigma_{i}^{(f)}\right)$. Then we have

LEMMA 1. Let notations be as above. Then

(a) $\beta_{i} \in B_{(i-1) f+1, \text { if }}$.

(b) $\beta_{i} \beta_{j}=\beta_{j} \beta_{i}$ for all $i, j \in \mathbf{N}$.

(c) $\sigma_{i}^{(f)}$ commutes with $\beta_{(n)}$ for all $n>i$.

(d) Similarly if $b \in \rho\left(k B_{f}\right)$, then $b_{i} \in \rho\left(k B_{(i-1) f+1, i f}\right)$ and $b_{(m)}$ commutes with $\rho^{(f)}\left(B_{n}\right)$ whenever $m>n$.

(e) If $\beta \in B_{f}$, there exists a well-defined automorphism $\Phi_{\beta}$ of $B_{\infty}$ such that if $\gamma \in B_{n f}, \Phi_{\beta}(\gamma)=\beta_{(n)}^{-1} \gamma \beta_{(n)}$.

Proof. (a) follows directly from the definition of the $\beta_{i}$ 's and (b) follows from (a) and relation (B2). By the remark after (4) conjugation by $\sigma_{i}^{(f)}$ permutes $\beta_{i}$ and $\beta_{i+1}$ and it leaves invariant $\beta_{j}, j \neq i, i+1$. This shows $(\mathrm{c})$. (d) follows from $(\mathrm{a})-(\mathrm{c})$ by linearity. 
If $\gamma \in B_{n f}$, it commutes with $\beta_{i}$ for $i>n$. It follows easily by induction on $m$ (using (a)) that

$$
\left(\beta_{(m)}\right)^{-1} \gamma \beta_{(m)}=\left(\beta_{(n)}\right)^{-1} \gamma \beta_{(n)} \text {. }
$$

Hence $\Phi_{\beta}$ is well defined. Obviously it is an automorphism whose restriction to $B_{n f}$ is inner for each $n \in \mathbf{N}$.

As the braid groups are torsion free, one can rescale a representation arbitrarily. More precisely this means if $\rho$ is a representation of $B_{\infty}$ and $\alpha \in k \backslash\{0\}$, then also the map $\sigma_{i} \mapsto \alpha \rho\left(\sigma_{i}\right)$ extends to a representation of $B_{\infty}$. Hence we will say that 2 representations $\rho$ and $\tilde{\rho}$ are (projectively) isomorphic denoted by $\rho \cong \tilde{\rho}$ if there exists a nonzero $\alpha \in k$ such that the map $\rho\left(\sigma_{i}\right) \mapsto \alpha \tilde{\rho}\left(\sigma_{i}\right), i \in \mathbf{N}$, can be extended to an isomorphism between $\rho\left(k B_{\infty}\right)$ and $\tilde{\rho}\left(k B_{\infty}\right)$.

In the following proposition, we are going to single out special subrepresentations of $\rho^{(f, \beta, \gamma)}$ determined by minimal idempotents of $\rho\left(k B_{f}\right)$. It will be shown that these subrepresentations are independent of the choice of $\beta$ and $\gamma$. This will enable us later to choose special $\beta$ 's and $\gamma$ 's for specific computations.

Proposition 2. Let $\rho$ be an $A F D$ representation of $B_{\infty}$.

(a) If $p \in \rho\left(k B_{f}\right)$ is an idempotent such that $\rho\left(k B_{n f}\right) p_{(m)} \cong$ $\rho\left(k B_{n f}\right) p_{(n)}$ for all $m>n$, then there exists an AFD representation $\rho^{(f, p)}$ of $B_{\infty}$ such that $\rho^{(f, p)}\left(k B_{n}\right) \cong \rho^{(f)}\left(k B_{n}\right) p_{(n)}$.

(b) If $p^{\prime}$ is another idempotent of $\rho\left(k B_{f}\right)$ which is equivalent to $p$, then we also have $\rho^{(f, p)} \cong \rho^{\left(f, p^{\prime}\right)}$.

(c) Let $\beta, \gamma \in B_{f}$ be such that $\rho(\beta)$ and $\rho(\gamma)$ are simultaneously diagonalizable. Then there exist mutually orthogonal minimal idempotents $p_{\mu}$ of $\rho\left(k B_{f}\right)$ adding up to 1 such that $\rho^{\left(f, p_{\mu}\right)} \cong \rho^{\left(f, \beta, \gamma, p_{\mu}\right)}$ for each of these minimal idempotents $p_{\mu}$.

(d) If $\rho$ is a unitary representation of $B_{\infty}$ on a complex Hilbert space $H$ and $p \in \rho\left(C B_{f}\right)$ is a selfadjoint projection, also $\rho^{(f, p)}$ is unitary.

Proof. (a) By Lemma 1, (d), $\sigma_{i} \in B_{n} \mapsto p_{(n)} \rho^{(f)}\left(\sigma_{i}\right)$ induces a representation of $B_{n}$ into $\rho\left(k B_{n f}\right)$. By assumption we have injections

$$
\rho^{(f)}\left(k B_{n}\right) p_{(n)} \rightarrow \rho^{(f)}\left(k B_{n+1}\right) p_{(n+1)} \rightarrow \rho^{(f)}\left(k B_{n+1}\right) p_{(n+2)} \rightarrow \cdots .
$$

We obtain a representation $\rho^{(f, p)}$ of $B_{\infty}$ into the inductive limit of these algebras by $\rho^{(f, p)}(\beta)=\rho^{(f)}(\beta) p_{(n)}$ for $\beta \in B_{n}$.

(b) Let $p^{\prime} \in \rho\left(k B_{f}\right)$ be an idempotent which is equivalent to $p$, i.e. there exists an invertible $b \in \rho\left(k B_{f}\right)$ such that $\tilde{p}=b p b^{-1}$. It 
is easy to check that $\tilde{p}_{(n)}=b_{(m)} p_{(n)} b_{(m)}^{-1}$ for all $m \geq n$. Moreover, we have an automorphism $\Phi_{b}$ on $\rho\left(k B_{\infty}\right)$ by $\Phi_{b}(a)=b_{(n)} a b_{(n)}^{-1}$ if $a \in \rho\left(k B_{n f}\right)$. It is easy to check that $\rho^{\left(f, p^{\prime}\right)}=\Phi_{b} \circ \rho^{(f, p)}$.

For (c), observe that $\rho(\beta)$ and $\rho(\gamma)$ generate a commutative semisimple finite dimensional algebra. Hence there exist minimal idempotents $\left(p_{\mu}\right)_{\mu \in I}$ (projections onto common 1-dimensional eigenspaces) and eigenvalues $\alpha_{1 \mu}$ and $\alpha_{2 \mu}$ of $\rho(\beta)$ and $\rho(\gamma)$ respectively such that $\rho(\beta) p_{\mu}=\alpha_{1 \mu} p_{\mu}$ and $\rho(\gamma) p_{\mu}=\alpha_{2 \mu} p_{\mu}$. Now observe that

$$
\begin{aligned}
\rho\left(\sigma_{i}^{(f, \beta, \gamma)}\right)\left(p_{\mu}\right)_{i}\left(p_{\mu}\right)_{i+1} & =\rho\left(\sigma_{i}^{(f)}\right) \rho\left(\beta_{i}\right)\left(p_{\mu}\right)_{i} \rho\left(\gamma_{i+1}\right)\left(p_{\mu}\right)_{i+1} \\
& =\alpha_{1 \mu} \alpha_{2 \mu} \rho\left(\sigma_{i}^{(f)}\right)\left(p_{\mu}\right)_{i}\left(p_{\mu}\right)_{i+1} .
\end{aligned}
$$

Hence, if $\alpha_{\mu}=\alpha_{1 \mu} \alpha_{2 \mu}$, we have by (2) $\rho^{\left(f, \beta, \gamma, p_{\mu}\right)}\left(\sigma_{i}\right)=\alpha \rho^{\left(f, \beta, p_{\mu}\right)}\left(\sigma_{i}\right)$ for all $i \in N$.

(d) follows immediately from the definitions.

REMARKS. 1. Observe that the direct sum of the subrepresentations $\rho^{\left(f, p_{\alpha}\right)}$ is only a subrepresentation of $\rho^{(f)}$.

2. It is also possible to decompose the complement of the subrepresentations of $\rho^{(f)}$ mentioned in the first remark. We will briefly sketch these results in the following, where we assume the representations to be unitary. For the known examples, this is enough as by analyticity arguments the results can also be extended to nonunitary representations (see next section).

Let $\tilde{p}_{n}$ be an idempotent of the form $p_{\mu_{1}, 1} p_{\mu_{2}, 2}, \ldots, p_{\mu_{n}, n}$ with $p_{\mu_{i}} \in \rho\left(k B_{f}\right)$ an idempotent of the partition of unity mentioned above and with $p_{\mu_{i}, i} \in \rho\left(k B_{(i-1) f+1, i f}\right)$ the corresponding 'shifted' idempotent. By definition of these idempotents, conjugation by a braid via $\rho^{(f)}$ just permutes the factors. Hence the centralizer $Z$ of $\tilde{p}_{n}$ is a subgroup of $B_{n}$ containing the pure braids. Let $\widetilde{P}_{(n)}$ be the sum of all conjugates of $\tilde{p}_{(n)}$. Then $\widetilde{P}_{(n)}$ is an idempotent invariant under $\rho^{(f)}\left(B_{n}\right)$ and by the imprimitivity theorem (see for instance [M], Theorem $(3.10))$, restriction of $\rho^{(f)}$ by $\widetilde{P}_{(n)}$ is isomorphic to the induction of $\rho_{\mid \tilde{p}_{(n)}}^{(f)}(Z)$ to $B_{n}$.

The importance of the braid groups for knot theory comes from the following fact: If the upper and the lower end of each string of the braid $\beta \in B_{n}$ are combined in the fashion indicated in Figure 3 (for $\left.\beta=\sigma_{1}^{3} \in B_{2}\right)$, one obtains the link $(\beta, n)^{\wedge}$ (see [B] or [BZ] for details). We have to keep track of the number of strings because the 

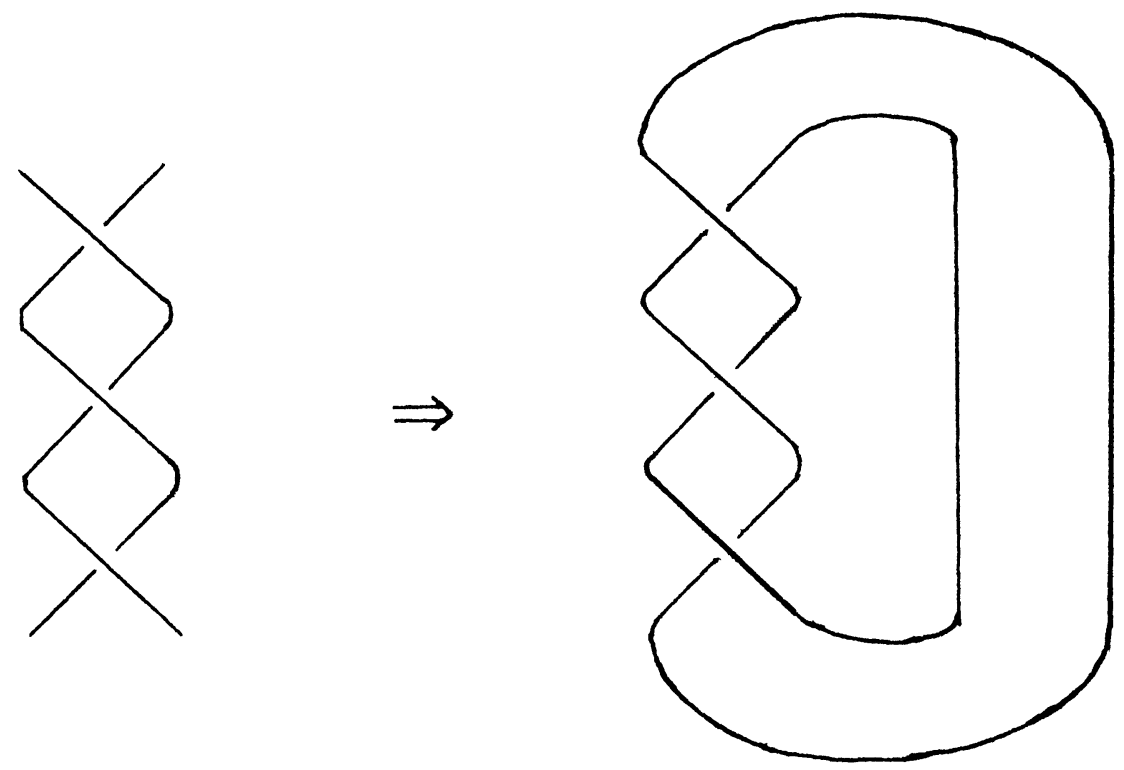

FIGURE 3

closure of $\beta \in B_{n}$, considered as an element of $B_{n+1}$ with respect to the usual embedding of $B_{n} \subset B_{n+1}$ yields a different link (see also [B] or $[\mathbf{J}-\mathbf{1}])$.

By a theorem of Alexander (see [B]) every link can be obtained in this way. There is an algorithm found by A. A. Markov which determines when 2 braids have the same closure. It can be described in the following way: 2 braids $\left(\beta_{1}, n_{1}\right)$ and $\left(\beta_{2}, n_{2}\right)$ have the same closure if one of them can be obtained from the other one after finitely many steps of the types

(M1) $(\beta, n) \leftrightarrow\left(\gamma^{-1} \beta \gamma, n\right)$ for $\gamma \in B_{n}$ and

(M2) $(\beta, n) \leftrightarrow\left(\sigma_{n}^{ \pm 1} \beta, n+1\right)$.

This can be used to find link invariants by special functionals on braid groups. As usual, a trace on an algebra $A$ is a functional from $A$ into its ground field such that $\operatorname{tr}(a b)=\operatorname{tr}(b a)$ for all $a, b \in A$. The annihilator ideal $I \subset A$ is the ideal defined by

$$
I=\{a \in A, \operatorname{tr}(a b)=0 \text { for all } b \in A\} .
$$

If $I=0$, the trace is said to be faithful (or nondegenerate).

If $A$ is a direct sum of full matrix algebras over $k$, this is equivalent to the fact that the restriction of tr to each of the simple components of $A$ is a nonzero multiple of the usual trace on matrix algebras, i.e. the sum of the diagonal elements. These multiples, which can be obtained by evaluating the trace at minimal idempotents of $A$, are referred to as weights of the trace. If all the weights are nonnegative, 
we say that the trace is positive. The following well-known observation follows from the fact that any minimal idempotent of a subalgebra $B \subset A$ can be written as a sum of minimal idempotents of $A$ :

If both $A$ and $B$ are direct sums of full matrix algebras and if $B \subset A$, the restriction of a positive, faithful trace on $A$ to $B$ is also positive and faithful.

By definition of $I$, there is a nondegenerate bilinear form on $A / I$ given by $\langle a, b\rangle=\operatorname{tr}(a b)$. Moreover, we have a representation $\rho_{\mathrm{tr}}$ of $A$ on $A / I$ given by left multiplication, the kernel of which is $I$. This procedure is known as Gelfand-Naimark-Segal (GNS) construction (see also $\S 3$ ).

The main ingredient for getting a link invariant via the approach of Jones is a Markov trace (see [J-1]) i.e. a trace $\operatorname{tr}$ on $k B_{\infty}$ such that for all $n \in \mathbf{N}$ and $\beta \in B_{n}$ we have

$$
\operatorname{tr}\left(\sigma_{n} \beta\right)=\operatorname{tr}\left(\sigma_{n}\right) \operatorname{tr}(\beta) .
$$

As conjugation by $\Delta_{n+1}$ maps $\sigma_{i}$ to $\sigma_{n+1-i}$ for $i=1,2, \ldots, n$ (see [B] or check it by pictures), (6) is equivalent to

$$
\operatorname{tr}\left(\sigma_{1} \beta\right)=\operatorname{tr}\left(\sigma_{1}\right) \operatorname{tr}(\beta) \quad \text { for all } \beta \in B_{2, \infty} .
$$

Note that after suitable rescaling (i.e replacing $\sigma_{i}$ by $\alpha \sigma_{i}$ for a suitably chosen $\alpha \in k$ ), we can always assume $\operatorname{tr}\left(\sigma_{i}\right)=\operatorname{tr}\left(\sigma_{i}^{-1}\right)$ for all $i \in \mathbf{N}$. In this case we say that $\operatorname{tr}$ is a normalized Markov trace. If 2 Markov traces have the same normalization in this sense, we will say that they are the same up to a scalar multiple. It follows from Markov's theorem (see $[\mathbf{J}-\mathbf{1}])$ that any normalized Markov trace defines a link invariant $L_{\text {tr }}$ by

$$
L_{\mathrm{tr}}\left((\beta, n)^{\wedge}\right)=\operatorname{tr}\left(\sigma_{1}\right)^{1-n} \operatorname{tr}(\beta) .
$$

Finally, we say that a Markov trace tr is multiplicative (or local), if

$$
\operatorname{tr}(\beta \gamma)=\operatorname{tr}(\beta) \operatorname{tr}(\gamma) \quad \text { for all } \beta \in B_{n}, \gamma \in B_{n+1, \infty} \text { and } n \in \mathbf{N} \text {. }
$$

Note that the Markov traces belonging to the 2-variable polynomial in [FYHLMO] and to the Kauffman polynomial are of this type. This can either be shown directly (see for instance [W-1], (3.1)) or by using Proposition 3 below.

We say that a link $K$ is the distant union of 2 links $K_{1}$ and $K_{2}$ if $K_{1}$ and $K_{2}$ can be separated by a plane (after possibly rearranging them). As usual the unknot is defined to be the closure of the braid (id, 1) 
which can be thought of as the boundary of a suitably embedded disc in 3-space. We can now formulate the easy converse of Jones' result.

Proposition 3. There exists a 1-1 correspondence between normalized multiplicative Markov traces with values in a field $k$ and $k$ valued link invariants $L$ with the properties

(a) $L($ unknot $)=1$ and

(b) there exists a fixed $z \in k \backslash\{0\}$ such that

$$
L\left(K_{1} \cup K_{2}\right)=z^{-1} L\left(K_{1}\right) L\left(K_{2}\right)
$$

for the distant union $K_{1} \cup K_{2}$ of arbitrary links $K_{1}$ and $K_{2}$. The correspondence is given by (8). One also has $z=\operatorname{tr}\left(\sigma_{i}^{ \pm 1}\right)$.

Proof. It is already shown that any normalized Markov trace defines a link invariant (see $[\mathbf{J}-\mathbf{1}]$ ). Property (a) is clear and property (b) follows from the multiplicativity of $\operatorname{tr}$. On the other hand if $\beta \in$ $B_{n}$, we have $(\beta, n+1)^{\wedge}=(\beta, n)^{\wedge} \cup$ unknot. This shows that the functional $\operatorname{tr}$ in (8) is well defined. It is a Markov trace by (M1) and (M2). Moreover, we also have

$$
1=L(\text { unknot })=L\left(\left(\sigma_{1}^{ \pm 1}, 2\right)^{\wedge}\right)=z^{-1} \operatorname{tr}\left(\sigma_{1}^{ \pm 1}\right) .
$$

This shows the last claim.

This observation already appeared implicitly in [BW], where it was used to construct representations of braid groups from Kauffman's link invariant. As a further application, it provides a tool for comparing link invariants by looking at the corresponding Markov traces and braid representations (see Theorem 8 ).

Let now tr be a Markov trace factoring over a representation $\rho$ of $B_{\infty}$. We will not distinguish in notation between $\operatorname{tr}$ and the trace it induces on $\rho\left(k B_{\infty}\right)$. If $b \in \rho\left(k B_{n f}\right)$ and $m>n$, we have $\operatorname{tr}\left(b p_{m}\right)=$ $\operatorname{tr}(b) \operatorname{tr}\left(p_{m}\right)$ by Lemma 1 and [W-1], $\S 3$. So whenever tr is faithful, we have $p_{(m)} \rho\left(k B_{n f}\right) p_{(m)} \cong p_{(n)} \rho\left(k B_{n f}\right) p_{(n)}$ for all $m>n$.

Let $\operatorname{tr}^{(p)}$ be the normalized trace which is obtained from $\operatorname{tr}$ by restriction to $\rho^{(f, p)}\left(B_{n}\right)$ and renormalization i.e. if $\beta \in B_{n}$, then $\operatorname{tr}^{(p)}(\beta)=1 /(\operatorname{tr}(p))^{n} \operatorname{tr}\left(\rho^{(f, p)}(\beta)\right)$. It is easy to see this restriction is well defined independently of $n$.

To get a link invariant we only need to prove that $\operatorname{tr}^{(p)}$ induces a Markov trace on $k B_{\infty}$ via $\rho^{(f, p)}$. We will give 2 different proofs for that. The first one follows Murakami's proof in [M]. 

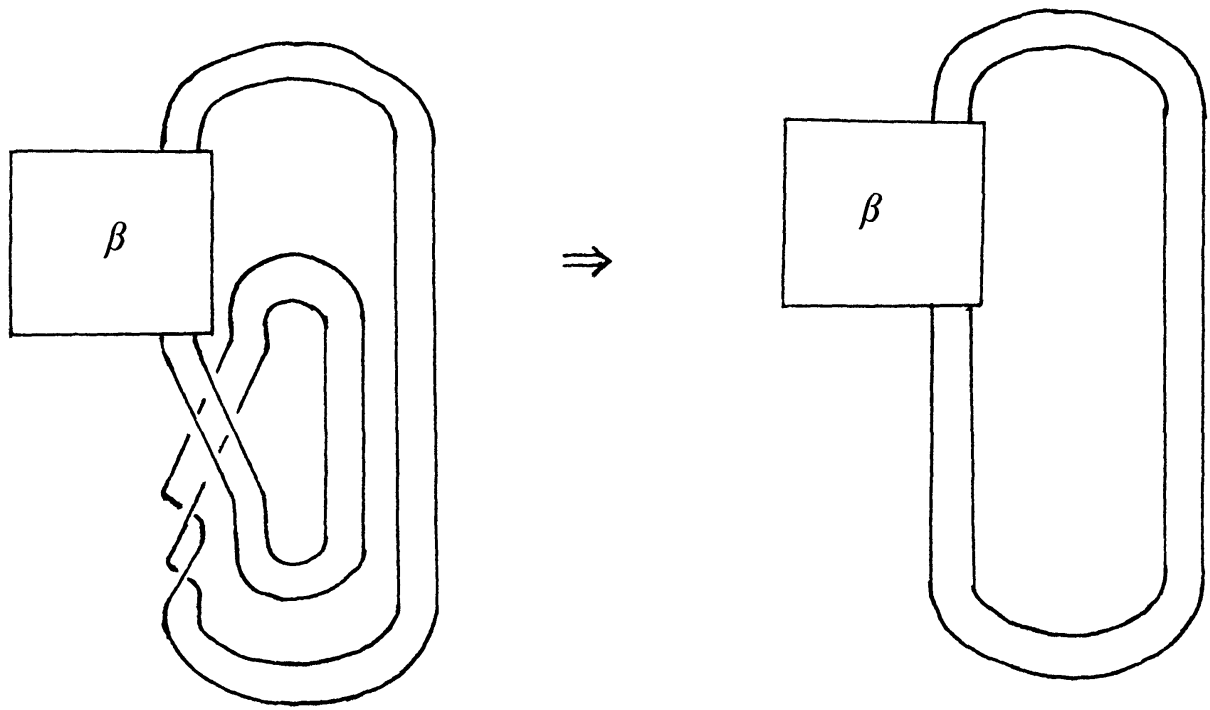

FIGURE 4

Proposition 4. Let tr be a multiplicative Markov trace and let $\rho=\pi_{\mathrm{tr}}$ be the representation coming from the GNS construction. Let $\tilde{\sigma}_{i}^{(f)}=\sigma_{i}^{(f)} \Delta_{(i-1) f+1, i f}^{-2}$.

(a) $\operatorname{tr}\left(\beta \tilde{\sigma}_{i}^{(f)}\right)=\operatorname{tr}(\beta) \operatorname{tr}\left(\tilde{\sigma}_{1}\right)^{f}$ for all $\beta \in B_{f+1, \infty}$.

(b) $\operatorname{tr}^{(f, p)}$ is a multiplicative Markov trace on $\rho^{(f, p)}\left(k B_{\infty}\right)$ for any idempotent $p \in \rho\left(k B_{f}\right)$.

(c) If $\beta, \gamma$ and $p_{\mu}$ are as in Proposition 2, one obtains a Markov trace from the representation $\rho^{\left(f, \beta, \gamma, p_{+} \mu\right)}$ as before. It coincides with $\operatorname{tr}^{(p)}$ up to scalar multiples if $p$ and $p_{\mu}$ are in the same simple component of $\rho\left(k B_{f}\right)$.

Proof. Let $L_{\mathrm{tr}}$ be the link invariant corresponding to $\operatorname{tr}$. It follows from the picture below that $\left(\beta \tilde{\sigma}_{j}^{(f)},(j+1) f\right)^{\wedge}$ and $(\beta, j f)^{\wedge}$ describe the same link.

Hence it follows from (8) that $\operatorname{tr}\left(\beta \tilde{\sigma}_{1}^{(f)}\right)=\operatorname{tr}(\beta) \operatorname{tr}\left(\tilde{\sigma}_{1}\right)^{f}$.

For the second statement note that $\Delta_{f}^{2}$ is in the center of $B_{f}$. With this it is shown, as before Lemma 1 , that $\rho\left(\tilde{\sigma}_{i}^{(f)}\right) p_{i+1}=p_{i} \rho\left(\tilde{\sigma}_{i}^{(f)}\right)$ and $\rho\left(\tilde{\sigma}_{i}^{(f)}\right) p_{i}=p_{i+1} \rho\left(\tilde{\sigma}_{1}^{(f)}\right)$. Hence

$$
p_{i} p_{i+1} \tilde{\rho}\left(\sigma_{i}^{(f)}\right) p_{i} p_{i+1}=p_{i} \rho\left(\tilde{\sigma}_{i}^{(f)}\right) p_{i} .
$$


Let $b \in \rho^{(f, p)}\left(k B_{i}\right) \subset p_{(i)} \rho\left(k B_{i f}\right) p_{(i)}$. Then it follows from the previous remark and (a)

$$
\operatorname{tr}\left(p_{(i+1)} b \rho\left(\tilde{\sigma}_{i}^{(f)}\right)\right)=\operatorname{tr}\left(b p_{i+1} \rho\left(\tilde{\sigma}_{i}^{(f)}\right)\right)=\operatorname{tr}\left(b \rho\left(\tilde{\sigma}_{i}^{(f)}\right)\right)=\operatorname{tr}(b) \operatorname{tr}\left(\tilde{\sigma}_{1}\right)^{f} .
$$

As $\operatorname{tr}^{(p)}$ is just a multiple of $\operatorname{tr}$, this shows that $\operatorname{tr}^{(f, p)}$ is a Markov trace. It follows from the definition and the multiplicativity of $\operatorname{tr}$ that it is also multiplicative.

The third claim follows from Proposition 2, (c).

2. Higher link invariants from the [FYHLMO] polynomial. We will now show at the example of the [FYHLMO] 2-variable polynomial that the Markov traces constructed by the procedure above correspond to new representations of braid groups. The representations of braid groups into Hecke algebras as well as other representations (e.g. the ones in [BW]) depend on one (or several) parameters. It is sometimes useful to consider those as representations over the field $k(x)$ of rational functions over $k$ where the parameter is replaced by the variable $x$. The following 2 lemmas relate the polynomial algebras over $k(x)$ to the concrete algebras with the parameter being an element of $k$.

Let $A$ be a finite dimensional $k(x)$ algebra and let $b_{1}, b_{2}, \ldots, b_{n}$ be a linear basis of $A$. Let $q \in k$ be such that the defining relations for $A$ also make sense if $x$ is replaced by $q$, i.e. $q$ is not a zero of the denominator of any rational function occurring in these relations. Then we can define the $k$ algebra $A(q)$ with the same basis $b_{1}, b_{2} \ldots, b_{n}$ and relations as above where the occurring rational functions are replaced by their values at $q$.

Observe that there is a ring homomorphism from $A$ onto $A(q)$ which maps the element $a=\sum_{i=1}^{n} \alpha_{i} b_{i} \in A$ to the element $a(q)=$ $\sum_{i=1}^{n} \alpha_{i}(q) b_{i} \in A(q)$. This evaluation homomorphism is well defined for all elements $a$ for which the coefficients $\alpha_{i}$ are well defined at $x=q$ for $i=1,2, \ldots, n$.

If $\operatorname{tr}$ is a trace on $A$, we obtain a (possibly not everywhere defined) trace $\operatorname{tr}_{q}$ on $A(q)$ by $\operatorname{tr}_{q}(a(q))=\operatorname{tr}(a)(q)$. We will in the following always assume that $\operatorname{tr}_{q}$ is well defined on $A(q)$.

If $a \in A, \operatorname{Tr}(a)$ is defined to be the trace of the linear operator on $A$ given by left multiplication by $a$. The discriminant $D_{A}\left(b_{1}, b_{2}, \ldots, b_{n}\right)$ is defined to be

$$
D_{A}\left(b_{1}, b_{2}, \ldots, b_{n}\right)=\operatorname{det}\left(\operatorname{Tr}\left(b_{i} b_{j}\right)\right) \text {. }
$$

It is well known that the fact whether $D_{A}\left(b_{1}, b_{2}, \ldots, b_{n}\right)$ is zero or not does not depend on the choice of the basis (see [Bk], $\S 9,5)$ and 
that this can be used as a criterion for the semisimplicity of $A$. Indeed if $A$ is not semisimple, we can choose $b_{1}$ to be in the radical of $A$. But then $b_{i} b_{1}$ is nilpotent for $i=1,2, \ldots, n$ and hence the first column of $\left(\operatorname{Tr}\left(b_{i} b_{j}\right)\right)$ only consists of zeros. On the other hand, if $A$ is a full matrix ring, the discriminant is not equal to 0 (see [Bk], $\S 9$, 5). In our context, any semisimple algebra is just a direct sum of full matrix algebras. Hence the discriminant is nonzero also in this case.

LEMMA 5. (a) Let $a_{1}, a_{2}, \ldots, a_{m}$ be linearly independent elements of $A$. Then $\left\{a_{1}(q), a_{2}(q), \ldots, a_{m}(q)\right\}$ is well defined and linearly independent for all but finitely many values of $q$.

(b) Let $B$ be a subalgebra of $A$. If $B(q)$ is semisimple for infinitely many values of $q$, then $B$ is semisimple and $\operatorname{dim}_{k} B(q)=\operatorname{dim}_{k(x)} B$ for all but finitely many values of $q$.

(c) Let $p \in A$ be an idempotent and let $q \in k$ such that $A(q)$ and $p(q)$ are well defined. Then $\operatorname{dim}_{k} B(q)=\operatorname{dim}_{k(x)} B$ for $B=p A p$.

(d) If there is a $q \in k$ such that $B(q)$ is semisimple and $\operatorname{dim}_{k} B(q)=$ $\operatorname{dim}_{k(x)} B$, then $B$ is semisimple. Moreover, in this case, we have for all but finitely many values of $q$ that $B(q)$ is well defined and $B(q) \otimes k(x) \cong B$.

(e) Let $\operatorname{tr}$ be a trace on $A$. If $\operatorname{tr}_{q}$ is faithful on $B(q)$ for some $q \in k$, then $\operatorname{tr}$ is faithful on $B$.

Proof. (a) Let $a_{i}=\sum_{j=1}^{n} c_{i j} b_{j}$ and let $C=\left(c_{i j}\right)$. As the $a_{i}$ 's are linearly independent, there exists an $m \times m$ minor $M$ of $C$ such that $\operatorname{det} M=\alpha \neq 0$. But then $a_{1}(q), a_{2}(q), \ldots, a_{m}(q)$ are well defined whenever $c_{i j}(q)$ is well defined for all entries of $C$ and they are linearly independent whenever $\alpha(q) \neq 0$.

(b) Let $a_{1}, a_{2}, \ldots, a_{m}$ be a basis for $B$. By (a) and assumption there exists a $q \in k$ such that $B(q)$ is semisimple and $a_{1}(q)$, $a_{2}(q), \ldots, a_{m}(q)$ is a basis for $B(q)$. Hence $D_{B}\left(a_{1}, \ldots, a_{m}\right) \neq 0$.

(c) is shown in [W-2], Lemma 2.3.

(d) We have $D_{B}\left(a_{1}, \ldots, a_{m}\right)(q)=D_{B}\left(a_{1}(q), \ldots, a_{m}(q)\right) \neq 0$. Hence $D_{B}\left(a_{1}, \ldots, a_{m}\right)$ is a nonzero rational function which has only a finite number of poles and zeros. For the last statement see for instance [W-2], Lemma 2.3.

(e) Let $a \in B$. By assumption, there exists a $b(q) \in B(q)$ such that $\operatorname{tr}_{q}(a(q) b(q))=z \neq 0$. So if $b \in B$ is an inverse image of $b(q)$ under the evaluation homomorphism, we have $\operatorname{tr}(a b)(q)=z \neq 0$.

Let now $\rho$ be a representation of $B_{\infty}$ over $k(x)$ such that $\rho\left(k(x) B_{n}\right)$ is finite dimensional for all $n \in \mathbf{N}$. If all matrix entries 
of $\rho\left(\sigma_{i}\right), i=1,2, \ldots, n$ are well defined at $x=q$ for all $n \in \mathbf{N}$, we obtain representations of $B_{n}$ over $k$. We denote the resulting representation of $B_{\infty}$ in the inductive limit by $\rho_{q}$. We can apply the lemma above in this setting where we take as a basis a maximum number of linearly independent images of group elements. One has the following consequences for $k=\mathbf{C}$.

LEMMA 6. Let $\rho$ be an AFD representation of $B_{\infty}$ over $\mathbf{C}(x)$ such that for each $n \in \mathbf{N}$ the restriction of $\rho_{q}$ to $B_{n}$ is unitarizable for infinitely many values of $q$.

(a) Then $\rho^{(p)}$ is an AFD representation of $B_{\infty}$ for any idempotent $p \in \rho\left(\mathbf{C}(x) B_{f}\right), f \in \mathbf{N}$.

(b) If $\operatorname{tr}$ is a trace on $\rho\left(\mathbf{C}(x) B_{n f}\right)$ such that its specialization $\operatorname{tr}_{q}$ on $\rho_{q}\left(\mathbf{C} B_{n f}\right)$ is faithful and positive for infinitely many values of $q$, then $\operatorname{tr}^{(p)}$ is faithful on $\rho^{(f, p)}\left(\mathbf{C}(x) B_{n}\right)$.

Proof. (a) Let $n \in \mathbf{N}$ and let $q \in \mathbf{C}$ such that $\rho_{q}$ can be made a unitary representation of $B_{n f}$. We can choose $p \in \rho\left(\mathbf{C} B_{n f}\right)$ such that $p(q)$ is a selfadjoint projection. Then $\left.\rho^{(f, p)}\right|_{B_{n}}$ is a finite dimensional unitary representation of $B_{n}$. The corresponding algebra is a finite dimensional $C^{*}$ algebra, hence semisimple. So the claim follows from Lemma 5, (d).

(b) By part (b) of the previous lemma and (a) $\rho_{q}^{(f, p)}\left(B_{n}\right)$ is well defined and semisimple for all but finitely many values of $q$. Hence it is so in particular for one of the values for which $\operatorname{tr}_{q}$ is positive and faithful. By (5), also the restriction of $\operatorname{tr}_{q}$ to $\rho_{q}^{(f, p)}\left(B_{n}\right)$ is faithful. So the claim follows from Lemma 5, (e).

Again we remark that these results could also be extended to the more general situation in the second remark after Proposition 4.

Let $H_{\infty}$ be the infinite Hecke algebra of type $A$ over $\mathbf{C}(x)$, the field of rational functions over $\mathbf{C}$, given by generators $g_{1}, g_{2}, \ldots$ and relations

(H1) $g_{i} g_{i+1} g_{i}=g_{i+1} g_{i} g_{i+1}$ for $i=1,2, \ldots$,

(H2) $g_{i} g_{j}=g_{j} g_{i}$, whenever $|i-j| \geq 2$,

(H3) $g_{i}^{2}=(x-1) g_{i}+x$ for $i=1,2, \ldots$.

If $q$ is a complex number, we denote by $H_{\infty}(q)$ the complex Hecke algebra generated by generators and relations as above where the variable $x$ is replaced by $q$. Similarly, we denote by $H_{n}$ resp. $H_{n}(q)$ the algebras generated by $g_{1}, g_{2}, \ldots, g_{n-1}$. It is well known that the dimension of $H_{n}$ (resp. $\left.H_{n}(q)\right)$ is $n$ !. 
If $q=1, g_{1}, g_{2}, \ldots, g_{n-1}$ satisfy the relations of a set of simple reflections of $S_{n}$. This can be used to find a natural basis for $H_{n}(q)$ in terms of reduced products of the $g_{i}$ 's, i.e. products for which the number of factors can no longer be reduced by applying (H3). With this basis the evaluation homomorphism from $H_{n}$ onto $H_{n}(q)$ is defined as above. It is known that except for $q$ a primitive $k$ th root of unity with $k=2,3, \ldots, n$ and $q=0, H_{n}(q) \cong \mathbf{C} S_{n}$, the group algebra of the symmetric group (see for instance [W-1], Theorem $(2,2)$ ). Similarly, $H_{n} \cong \mathbf{C}(x) S_{n}$ which could, for instance, be proved using Lemma 5.

So whenever $H_{n}(q)$ is semisimple, all its irreducible representations up to conjugacy are labelled by the elements of the set $\Lambda_{n}$ of all Young diagrams with $n$ boxes. Recall that a Young diagram $\lambda \in \Lambda_{n}$ is given by an $m$-tuple $\left[\lambda_{1}, \lambda_{2}, \ldots, \lambda_{m}\right]$ such that $\lambda_{1} \geq \lambda_{2} \geq \cdots \geq \lambda_{m} \geq 0$ and $\lambda_{1}+\lambda_{2}+\cdots+\lambda_{m}=n$. We will denote by $\pi_{\lambda}$ the irreducible representation of $H_{n}(q)$ belonging to $\lambda \in \Lambda_{n}$ as defined in [W-1], $\S 2$. Similarly, we have $H_{n} \cong \mathbf{C}(x) S_{n}$.

One can define for each standard tableau $t$ with $n$ boxes a minimal idempotent $p_{t} \in H_{n}$ such that their sum adds up to 1 (see [W-1], Cor. (2.3)). The idempotent $p_{t}(q) \in H_{n}(q)$ is a well-defined minimal idempotent whenever $H_{n}(q)$ is semisimple. See also [G] for a different set of minimal idempotents of $H_{n}(q)$.

Let $p \in H_{n}$ be an idempotent such that $p(1)$ is well defined. In particular, as $H_{n}(1) \cong \mathbf{C} S_{n}$ is semisimple and $H_{n} \cong \mathbf{C}(x) \otimes \mathbf{C} S_{n}$, it follows from Lemma 5

$$
p H_{n} p \cong \mathbf{C}(x) \otimes p(1) H_{n}(1) p(1)
$$

So in many cases the structure of certain subalgebras of $H_{n}$ or $H_{n}(q)$ can be determined from information about $H_{n}(1)=\mathbf{C} S_{n}$.

We will use this observation in the following context: Let $V$ be a vector space of dimension $\geq 2 f$ and let $V^{\otimes 2 f}$ be the $2 f$-fold tensor product. It is known that the permutation of factors in $V^{\otimes 2 f}$ induces a faithful representation of $\mathbf{C} S_{2 f}$. By Schur-Weyl duality, each minimal idempotent of $\mathrm{CS}_{2 f}$ corresponds to an irreducible representation of $\mathrm{Gl}(V)$. If $p_{1}(1) \in H_{f}(1) \cong \mathbf{C} S_{f}$ is a minimal idempotent belonging to the Young diagram $\lambda$, we therefore obtain the decomposition of $p_{1}(1) p_{2}(1)$ into a sum of minimal idempotents of $\mathbf{C} S_{2 f}$ from the corresponding decomposition of $\rho_{\lambda} \otimes \rho_{\lambda}(\mathrm{Gl}(V))$ into irreducible representations. This is well known by the Littlewood-Richardson rule. 
If $\lambda=[f]$, we have (see $[\mathbf{M c}])$

$$
\rho_{[f]} \otimes \rho_{[f]}=\bigoplus_{i=0}^{f} \rho_{[f+i, f-i]} .
$$

Hence there are minimal idempotents $p_{[f+i, f-i]}(1) \in H_{[f+i, f-i]}(1)$ for $i=0,1, \ldots, f$ such that

$$
p_{1}(1) p_{2}(1)=\sum_{i=0}^{f} p_{[f+i, f-i]}(1) .
$$

By (1) and Lemma 5 we obtain the same decomposition if $p_{1}$ is the central idempotent in $H_{n}$ which corresponds to [f], namely there exist minimal idempotents $p_{[f+i, f-1]} \in H_{n}, i=0,1, \ldots, f$ such that

$$
p_{1} p_{2}=\sum_{i=0}^{f} p_{[f+i, f-i]} .
$$

If $q$ is a primitive $l$ th root of unity and $k=1,2, \ldots, l-1$, there also exist semisimple representations $\pi_{\lambda}^{(k, l)}$ of $H_{n}(q)$ for each $(k, l)$ diagram $\lambda$ with $n$ boxes. By this we mean a Young diagram with at most $k$ rows such that $\lambda_{1}-\lambda_{k} \leq l-k$. If $q=e^{ \pm 2 \pi i / l}$ these representations are unitary, i.e. $\pi_{\lambda}^{(k, l)}\left(g_{i}\right)$ is a unitary matrix for $i=1,2, \ldots, n-1$ (see [W-1], Prop. 2.10). We thereby obtain for this special value of $q$ a representation $\pi^{(k, l)}$ of $H_{\infty}(q)$ such that

$$
\pi^{(k, l)}\left(H_{n}(q)\right) \cong \bigoplus_{\lambda \in \Lambda_{n}^{(k, l)}} \pi_{\lambda}^{(k, l)}\left(H_{n}(q)\right) .
$$

It follows immediately from (H1) and (H2) that $\sigma_{i} \mapsto g_{i}$ defines a homomorphism of $B_{\infty}$ into $H_{\infty}(q)$ (or $B_{n}$ into $H_{n}(q)$ respectively). Hence the representations of the Hecke algebra mentioned before can be lifted to representations of the braid groups which will also be denoted by $\pi_{\lambda}$ and $\pi_{\lambda}^{(k, l)}$ respectively.

Note that $(\mathrm{H} 3)$ can be written as $\left(g_{i}-x\right)\left(g_{i}+1\right)=0$. Hence the element

$$
e_{i}=\frac{x-g_{i}}{x+1}
$$

can be interpreted as spectral projection (or eigen projection) belonging to the eigenvalue -1 of $g_{i}$. It follows from work of Ocneanu (see [O], [J-1] or [W-1]) that (normalized) Markov traces factoring over representations of $B_{\infty}$ into $H_{\infty}(q)$ are uniquely determined by $q$ 
and the additional parameter $\eta=\operatorname{tr}\left(e_{1}\right)$. We will denote the Markov trace given by these parameters by $\operatorname{tr}_{q, \eta}$. Another way of stating this result is that there exists a trace $\operatorname{tr}=\operatorname{tr}_{x, \nu}$ on $H_{\infty}$ with values in the field $\mathbf{C}(x, \nu)$ of rational functions in 2 variables such that $\operatorname{tr}(a)(q, \eta)=\operatorname{tr}_{q, \eta}(a(q))$.

Let us define for $k \in \mathbf{Z} \backslash\{0\}$ the rational function

$$
a_{k}(x)=\frac{1-x^{k+1}}{(1+x)\left(1-x^{k}\right)} .
$$

By [W-1], Theorem 3.6, the representations $\pi^{(k, l)}$ are isomorphic to the representation coming from the GNS construction with respect to the Markov trace $\operatorname{tr}_{q, \eta}$ with parameters $q=e^{ \pm 2 \pi i / l}$ and $\eta=a_{-k}(q)$. Moreover, the trace is a positive trace on the image of $\pi_{\mathrm{tr}}$. Note that if $l>n$ any Young diagram $\lambda \in \Lambda_{n}$ is a $(k, l)$ diagram. Hence $\operatorname{tr}$ is a positive and faithful trace on $H_{n}(q)$.

By Proposition 4 we obtain for a minimal idempotent $p$ in $\pi_{\lambda}\left(H_{n}(q)\right)$ a representation $\rho^{(\lambda)}$ of $B_{\infty}$. We intend to show that the representations $\rho^{(f, p)}$, where $p$ is the idempotent belonging to the diagram $[f]$ are mutually nonisomorphic for all $f \in \mathbf{N}$.

Let $r_{\lambda}$ be the rank of $\pi_{\lambda}\left(e_{i}\right)$. Moreover, let $d_{\lambda}$ be the degree of $\pi_{\lambda}$. Obviously, $l_{\lambda}=d_{\lambda}-r_{\lambda}$ is the rank of the eigenspace of $\pi_{\lambda}\left(g_{i}\right)$ belonging to the eigenvalue $x$. Note that the representation $\pi_{\lambda}$ of $H_{n}$, restricted to $H_{n-1}$ decomposes into a direct sum of representations $\pi_{\lambda^{\prime}}$ belonging to all those diagrams $\lambda^{\prime}$ which can be obtained by taking away a box from $\lambda$. We will write this as

$$
\pi_{\lambda \mid H_{n-1}} \cong \bigoplus_{\lambda^{\prime}<\lambda} \pi_{\lambda^{\prime}}
$$

It follows from this

$$
d_{\lambda}=\sum_{\lambda^{\prime}<\lambda} d_{\lambda^{\prime}}
$$

and

$$
r_{\lambda}=\sum_{\lambda^{\prime}<\lambda} r_{\lambda^{\prime}}
$$

By definition of the representations in $[\mathbf{W}-\mathbf{1}]$, we have $d_{[f]}=1$ and $\pi_{[f]}\left(e_{1}\right)=0$ for all $f \in \mathbf{N}$. Hence $r_{[f]}=0$ and $r_{[1,1]}=1$. It is now easy to show by induction on $n$, the number of boxes of a diagram, that

$$
r_{\left[\lambda_{1}-1, \lambda_{2}-1\right]}=d_{\left[\lambda_{1}-1, \lambda_{2}-1\right]}
$$

for any diagram $\lambda=\left[\lambda_{1}, \lambda_{1}\right]$ such that $\lambda_{2} \geq 1$. 
LemMA 7. Let $\lambda \in \Lambda_{j}$. Then

(a) $\operatorname{det}\left(\pi_{\lambda}\left(\sigma_{i}\right)\right)=x^{l_{\lambda}}(-1)^{r_{\lambda}}$.

(b) $\pi_{\lambda}\left(\Delta_{f}^{2}\right)=x^{f(f-1) l_{\lambda} / d_{\lambda}} 1 V_{\lambda}$.

(c) If $\lambda=\left[\lambda_{1}, \lambda_{2}\right]$, then $\pi_{\lambda}\left(\Delta_{f}^{2}\right)=x^{f(f-1)-\left(\lambda_{1}+1\right) \lambda_{2}} 1_{V_{\lambda}}$.

Proof. Part (a) is clear. For part (b) just note that $\Delta_{f}^{2}$ consists of $f(f-1)$ factors all of which are conjugate to $g_{1}$. Hence $\operatorname{det}\left(\pi_{\lambda}\left(\Delta_{f}^{2}\right)\right)=$ $x^{f(f-1) l_{\lambda}}$. But as $\pi_{\lambda}$ is irreducible and as $\Delta_{f}^{2}$ is in the center of $B_{f}$, we have

$$
\pi_{\lambda}\left(\Delta_{f}^{2}\right)=x^{f(f-1) l_{\lambda} / d_{\lambda}} 1_{V_{\lambda}} .
$$

For (c) note that if $\lambda=\left[\lambda_{1}, \ldots, \lambda_{k}\right]$, the degree $d_{\lambda}$ is given by the formula (see for instance [R], p. 44)

$$
d_{\lambda}=n ! \frac{\prod_{1 \leq i<j \leq k} \lambda_{i}-\lambda_{j}+j-i}{\prod_{i=1}^{k}\left(\lambda_{i}+k-i\right) !}
$$

If $\lambda=\left[\lambda_{1}, \lambda_{2}\right]$, we obtain from this and (15)

$$
f(f-1) r_{\lambda} / d_{\lambda}=\left(\lambda_{1}+1\right) \lambda_{2} .
$$

Using $l_{\lambda}=d_{\lambda}-r_{\lambda}$, the claim follows from the last equation and (b).

THEOREM 8. There exists for each Young diagram $\lambda$ with $f$ boxes an AFD representation $\rho^{(\lambda)}$ of $B_{\infty}$ over the field $\mathbf{C}(x)$ of rational functions over C. Moreover there exists a Markov trace $\operatorname{tr}^{(\lambda)}$ with values in $\mathbf{C}(x, \nu)$ such that $\pi_{\mathrm{tr}} \cong \rho$. In particular, we obtain for each Young diagram $\lambda$ as above a 2-variable link invariant $L_{\lambda}$.

In case of the Young diagram with one box, the corresponding representation is the Hecke algebra representation of the braid group and the link invariant is the 2-variable polynomial of [FYHLMO]. Moreover, one also has:

(a) Let $\lambda^{*}$ be the Young diagram having $\lambda_{1}$ boxes in its first column, $\lambda_{2}$ boxes in its second column etc. and let $V$ be a link. Then $L_{\lambda^{*}}(V)$ can be obtained by replacing every occurrence of $x$ and $\nu$ in $L_{\lambda}(V)$ by $x^{-1}$ and $1-\nu$ respectively.

(b) Let $\rho^{([f])}$ be the representation corresponding to the Young diagram $[f]$. Then $\rho^{([f])}\left(\sigma_{i}\right)$ has exactly $f+1$ distinct eigenvalues, which are up to multiplication by a constant power of $x$ contained in $\left\{ \pm x^{f(2 f-1)-(f+i+1)(f-i) / 2}, i=0,1, \ldots, f\right\}$. In particular, $\rho^{([f])} \not$ $\rho^{([h])}$ for $f \neq h$. 
(c) Let $q=e^{ \pm 2 \pi i / l}$. There exists for each $(k, l)$ diagram $\lambda \in \Lambda_{f}^{(k, l)}$ a unitary representation $\rho^{(k, l, \lambda)}$ of $B_{\infty}$ into a quotient of $H_{\infty}(q)$ such that $\rho^{(k, l,[1])}=\pi^{(k, l)}$.

Proof. Let $\rho$ be a minimal idempotent in $\pi_{\lambda}\left(H_{f}\right)$. Then we can construct a new representation of $B_{\infty}$ by Proposition 3. By Lemma 2 this representation does not depend on the choice of $p$ so that we can simply label it by $\rho^{(\lambda)}$.

As mentioned above the Markov trace $\operatorname{tr}=\operatorname{tr}_{q, \eta}$ is faithful and positive on $H_{n f}(q)$ for $q=e^{2 \pi i / l}$ with $l>2 n f$ and $\eta=a_{n f}(q)$ and the corresponding representation $\pi_{\mathrm{tr}}$ yields a unitary representation of $B_{n f}$. Hence also $\rho^{(\lambda)}$ is an AFD representation and $\operatorname{tr}^{(\lambda)}$ is faithful on $\rho^{(\lambda)}\left(\mathbf{C}(x) B_{n}\right)$ by Lemma 6.

For (a) let $g_{i}\left(x^{-1}\right), i=1,2, \ldots, n-1$ be the standard generators of $H_{n}\left(x^{-1}\right)$. It is easy to check that the map $\Phi: g_{i}\left(x^{-1}\right) \mapsto$ $-x^{-1} g_{i}$ extends to an isomorphism between $H_{n}\left(x^{-1}\right)$ and $H_{n}$ mapping $e_{i}\left(x^{-1}\right)$ (the spectral projection of $g_{i}\left(x^{-1}\right)$ belonging to -1 ) to $1-e_{i}$. As noted before, any Markov trace on $H_{n}\left(x^{-1}\right)$ is uniquely determined by the value of $\nu=\operatorname{tr}\left(e_{1}\right)$. It follows from this that $\operatorname{tr}_{r, \nu} \circ \Phi$ is equal to the Markov trace $\operatorname{tr}_{x^{-1}, 1-\nu}$. The claim now follows as soon as it is shown that $\pi_{\lambda}\left(\Phi\left(H_{n}\left(x^{-1}\right)\right)=\pi_{\lambda^{*}}\left(H_{n}\right)\right.$. This fact is well known for $H_{n}(1)=\mathbf{C} S_{n}$. The general statement now follows from the evaluation homomorphism and (10) (with $z_{\lambda^{*}}$, the central idempotent belonging to $\pi_{\lambda^{*}}$ for $p$ ).

(b) Let $p=p_{1}$ be the minimal projection corresponding to the 1-dimensional representation of $H_{n}$ belonging to $[f]$. By Proposition 2 and the remarks before (7), the representations $\rho^{(f, p)}$ and $\rho^{\left(f, p, \Delta_{f}, \Delta_{f}\right)}$ are equivalent up to scalar multiples. Moreover, observe that

$$
\begin{aligned}
\rho^{\left(f, p, \Delta_{f}, \Delta_{f}\right)}\left(\sigma_{i}\right) & =\rho\left(\sigma_{i}^{(f)} \Delta_{(i-1) f+1, i f} \Delta_{i f+1,(i+1) f}\right) \\
& =\rho\left(\Delta_{(i-1) f+1,(i+1) f}\right) .
\end{aligned}
$$

Hence the spectral values of $\rho^{[f]}\left(\sigma_{1}\right)$ can be obtained from the ones of $p_{1} p_{2} \rho\left(\Delta_{2 f}\right)$ up to a constant multiple.

Let $p_{[f+i, f-i]}, i=0,1, \ldots, f$ be the minimal idempotents of $H_{2 f}$ occurring in the decomposition of $p_{1} p_{2}$ in (11). Recall that $\Delta_{2 f}^{2}$ is in the center of $B_{2 f}$. Hence it follows from Lemma 7 that $\rho\left(\Delta_{2 f}\right)^{2} p_{[f+i, f-i]}=x^{2 f(2 f-1)-(f+1+i)(f-i)} p_{[f+i, f-i]}$. As $\rho\left(\Delta_{2 f}\right)$ 
commutes with $p_{1} p_{2}$, we have

$$
\left.\rho\left(\Delta_{2 f}\right) p_{[f+i, f-i]}=\mu p_{[f+i, f-i]} \quad \text { with } \mu \in \pm x^{f(2 f-1)-(f+1+i)(f-i) / 2}\right\} .
$$

Observe that the function $t \mapsto(f+1+t)(f-t)$ is strictly decreasing for $t \geq 0$. Hence $\rho\left(\Delta_{2 f}\right)=\rho^{[f]}\left(\sigma_{1}\right)$ has exactly $f+1$ distinct spectral values and $\rho^{[f]} \not \rho^{[h]}$ for $f \neq h$.

The last statement follows immediately from Proposition 2, (d)

REMARKS 1 . Similar results have been independently obtained by J. Murakami. In his paper [M], one can also find explicit computations of invariants for many links.

2. An analogous result can be obtained for the algebras in [BW]. More precisely, we can again define for each Young diagram a 2variable link invariant corresponding to the diagram with one box is the Kauffman polynomial. This algebra will be studied in detail in [W-3].

3. If one sets $\eta=a_{-k}(q)$, the corresponding one variable invariants can also be obtained directly using the quantum group $U_{q}\left(s l_{k}\right)$ (see [Re], [Ro] and §4). For special cases, this was also shown in [AW].

3. Unitarity properties. Our original proof of Proposition 4 used a variation of Weyl's 'unitarian trick'. We exploit the fact that the 2 variable polynomial is already determined if one knows its values for $q=e^{2 \pi i / l}$ and $\eta=a_{k}(q)$ for $k=1,2, \ldots, l-1$. For these values one can use positivity arguments to prove the existence and additional properties of these link invariants. We will need the GNS construction in its form for Hilber $r_{\imath}$ spaces. Any trace on a complex algebra $A$ with an involution * (i.e a conjugate linear map $a \mapsto a^{*}$ such that $\left(a^{*}\right)^{*}=a$ and $\left.(a b)^{*}=b^{*} a^{*}\right)$ induces a sesquilinear form on $A$ by $\langle a, b\rangle=\operatorname{tr}\left(b^{*} a\right)$. Factoring over the annihilator ideal $I=\{a \in$ $A, \operatorname{tr}(a b)=0$ for all $b \in A\}$, we obtain a nondegenerate sesquilinear form on $\bar{A}=A / I$. Let $\pi_{\mathrm{tr}}$ be the representation of $A$ on $\bar{A}$ defined by left multiplication, i.e. $\pi_{\mathrm{tr}}(a) \bar{b}=\overline{a b}$.

We will need the following assumptions: Let $A=\mathbf{C} B_{\infty}$ and let $\operatorname{tr}$ be a trace on $A$ which defines a positive definite sesquilinear form on $\bar{A}$. Then $\bar{A}$ can be completed to a Hilbert space $H$. We also assume that $\pi_{\mathrm{tr}}$ is a unitary representation of $B_{\infty}$ on $H$. Let $L(H)$ denote the set of all bounded linear operators on $H$ and let for any algebra $C$ of linear operators on $H$ the set of commutants be denoted by $C^{\prime}$, i.e.

$$
C^{\prime}=\{d \in L(H), d c=c d \text { for all } c \in C\} .
$$


As $\operatorname{tr}$ is a finite trace, $M=\pi_{\mathrm{tr}}\left(\mathbf{C} B_{\infty}\right)^{\prime \prime}$ is a finite von Neumann algebra on $H$. With these notations the trace $\operatorname{tr}$ is called a factor trace if $M$ is a factor, i.e. the only central elements in $M$ are multiples of the identity. It follows from [W-1], Proposition 3.2 that

Any positive multiplicative Markov trace tr on $C B_{\infty}$ is a factor trace.

Recall that there exists for any sub-von Neumann algebra $N \subset M$ a conditional expectation $\varepsilon_{N}: M \rightarrow N$, defined in the following way. If $a \in M$, the image $\varepsilon_{N}(a)$ is the unique element in $N$ such that

$$
\operatorname{tr}(a b)=\operatorname{tr}\left(\varepsilon_{N}(a) b\right) \quad \text { for all } b \in N .
$$

If $p \in M$ is a projection, we define $M_{p}=\{$ pap, $a \in M\}$. Recall that if $M$ is a factor, so is $M_{p}$. With these notations we have

Lemma 9. Let $A \subset B \subset C$ be $\mathrm{II}_{1}$ factors such that $A^{\prime} \cap B=\mathrm{C} 1$. Let $b \in B$ and $c \in A^{\prime} \cap C$. Then

$$
\operatorname{tr}(b c)=\operatorname{tr}(b) \operatorname{tr}(c) \text {. }
$$

Proof. Using (17), it is checked easily that $\varepsilon_{B}\left(A^{\prime} \cap C\right)=A^{\prime} \cap B=\mathbf{C} 1$. Hence $\varepsilon_{B}(c)=\operatorname{tr}(c) 1$ and again by $(17)$

$$
\operatorname{tr}(b c)=\operatorname{tr}\left(b \varepsilon_{B}(c)\right)=\operatorname{tr}(b) \operatorname{tr}(c) .
$$

If $q=e^{ \pm 2 \pi i / l}$ and $\eta=a_{-k}(q)$, the representation $\pi_{\mathrm{tr}}$ with respect to the Markov trace $\operatorname{tr}=\operatorname{tr}_{q, \eta}$ given by these parameters is unitary and $\pi_{\mathrm{tr}}\left(B_{\infty}\right)^{\prime \prime}$ is isomorphic to the hyperfinite $\mathrm{II}_{1}$ factor. It has been shown in [W-2], Theorem (3.7) that in this case

$$
\pi_{\mathrm{tr}}\left(B_{m+1, \infty}\right)^{\prime} \cap \pi_{\mathrm{tr}}\left(\mathbf{C} B_{m}\right) \quad \text { for all } m \in \mathbf{N} \text {. }
$$

Theorem 10. Let $n \in \mathbf{N}, \beta_{1} \in B_{n-1}, \beta_{2} \in B_{n, \infty}$ and let $\lambda \in \Lambda_{f}$. Then, if $\operatorname{tr}^{(\lambda)}$ is as in Theorem 8 , we have

$$
\operatorname{tr}^{(\lambda)}\left(\beta_{1} \beta_{2}\right)=\operatorname{tr}^{(\lambda)}\left(\beta_{1}\right) \operatorname{tr}^{(\lambda)}\left(\beta_{2}\right)
$$

Proof. Let $t$ be a standard tableau belonging to the Young diagram $\lambda$ with $f$ boxes and let $q=e^{2 \pi i / l}$ with $f>m$. As $H_{f}(q)$ is semisimple, the minimal idempotent $p_{t}(q)$ is well defined for each standard tableau $t$ with $f$ boxes (see text above or [W-1], Corollary 2.3). In particular, we can define for $p=p_{t}(q) \in \pi_{\mathrm{tr}}\left(\mathbf{C} B_{f}\right)$ the projections $p_{1}$, $p_{2}, p_{(n)}$, etc. as before. If $\eta=a_{-k}(q)$ with $k>f, \operatorname{tr}_{q, \eta}\left(p_{t}(q)\right)=0$ if and only if $\lambda$ contains more than $k$ rows. 
Let $\pi_{\mathrm{tr}}$ be the representation coming from the GNS construction with respect to $\operatorname{tr}$ and let $m \in \mathbf{N}$ such that $\beta_{1} \beta_{2} \in B_{m}$. By the remarks above we obtain factors

$$
\begin{aligned}
A & =p_{(m)} \pi_{\operatorname{tr}}\left(B_{(n+1) f+1, \infty}\right)^{\prime \prime} p_{(m)} \subset B=p_{(m)} \pi_{\operatorname{tr}}\left(B_{n f+1, \infty}\right)^{\prime \prime} p_{(m)} \subset C \\
& =p_{(m)} \pi_{\operatorname{tr}}\left(B_{\infty}\right)^{\prime \prime} p_{(m)} .
\end{aligned}
$$

As $p$ is a minimal projection of $\pi_{\mathrm{tr}}\left(B_{f}\right)^{\prime \prime}$, it follows from (18) that $A^{\prime} \cap B=\mathrm{C} 1$. Let $\operatorname{tr}$ be the normalized trace on $C$. Observe that

$$
\tilde{\operatorname{tr}} r\left(p_{(m)} \pi_{\mathrm{tr}}^{(f)}(\beta)\right)=\operatorname{tr}^{(\lambda)}(\beta) \quad \text { for all } \beta \in B_{m}
$$

and that $p_{(m)} \pi_{\mathrm{tr}}^{(f)}\left(\beta_{1}\right) \in A^{\prime} \cap C$. But then the claim follows for our special choice of parameters $q=e^{2 \pi i / l}$ and $\eta=a_{-k}(q)$ from this and the previous lemma.

Observe that both sides in $(*)$ consist of rational functions. Hence it also holds for arbitrary $q$ by the principle of isolated zeros. But then, we also have for a given $q \in \mathbf{C}$ which is not a root of unity that the statement is true for infinitely many values $\eta=a_{k}(q), k \mathbf{Z} \backslash\{0\}$. From this follows the claim for arbitrary $\eta$ as before.

COROLLARY. Let $L^{(\lambda)}$ be the link invariant corresponding to $\operatorname{tr}^{(\lambda)}$ and let $K_{1}, K_{2}$ be knots. Then

(a) $L^{(\lambda)}\left(\beta \sigma_{n}^{m}, n+1\right)^{\wedge}=\left(\operatorname{tr}^{(\lambda)}\left(\sigma_{n}^{(m)}\right) / \operatorname{tr}^{(\lambda)}\left(\sigma_{n}\right)\right) L^{(\lambda)}(\beta, n)^{\wedge}$.

(b) $L^{(\lambda)}\left(K_{1} \# K_{2}\right)=L^{(\lambda)}\left(K_{1}\right) L^{(\lambda)}\left(K_{2}\right)$.

Proof. The proof of the first statement follows immediately from the theorem and (8). For (b) just observe that if $\left(\beta_{1}, n_{1}\right)^{\wedge}=K_{1}$ and $\left(\tilde{\beta}_{2}, n_{2}\right)^{\wedge}=K_{2}$, we have

$$
K_{1} \# K_{2}=\left(\beta_{1} \sigma_{n_{1}}, \beta_{2}, n_{1}+n_{2}\right)^{\wedge},
$$

where $\beta_{2}$ is the image of $\tilde{\beta}_{2}$ under the shift induced by $\sigma_{i} \rightarrow \sigma_{i+n_{1}}$.

Remark. By Proposition 2, (d) and (16), one obtains the subfactor $\pi_{\mathrm{tr}^{(\lambda)}}\left(B_{2, \infty}\right)^{\prime \prime} \subset \pi_{\mathrm{tr}^{(\lambda)}}\left(B_{\infty}\right)^{\prime \prime}$ for $q=e^{2 \pi i / l}$ and $\eta=a_{-k}(q)$. It seems likely that it is isomorphic to the subfactor $p \pi_{\mathrm{tr}}\left(B_{f+1, \infty}\right)^{\prime \prime} \subset$ $p \pi_{\mathrm{tr}}\left(B_{\infty}\right)^{\prime \prime} p$, where $p$ is a minimal idempotent of $\pi_{\lambda}\left(H_{f}(q)\right)$. A proof of this seems complicated, however, as little is known about the algebras $\pi_{\operatorname{tr}^{(\lambda)}}\left(\mathbf{C} B_{f}\right)$.

4. Quantum Yang-Baxter equations. In his paper [Ji] (see also Drinfeld's paper [D] for another approach) M. Jimbo constructed for each classical Lie algebra in its standard representation on a vector space $V$ 
explicit solutions of the quantum Yang-Baxter equations (QYBE). He thus obtained matrices $R(x)$ on $V \otimes V$, depending on a parameter $x$ and an additional parameter $q$ with the following property: Let $R_{i j}(x)$ be the matrix acting on $V \otimes V \otimes V$ as $R(x)$ on the $i$ th and $j$ th copy of $V$ and as the identity on the remaining one. Then we have

$$
R_{12}(x) R_{13}(x y) R_{23}(y)=R_{23}(y) R_{13}(x y) R_{12}(x) .
$$

Let moreover $P_{i j}$ be the permutation between the $i$ th and the $j$ th factor in $\otimes^{\infty} V$ and let $R_{i j}^{\vee}=P_{i j} R_{i j}$. Then we obtain the following well-known transformation of the QYBE:

$$
\begin{aligned}
R_{12}^{\vee}(y) R_{23}^{\vee}(x y) R_{12}^{\vee}(x) & =\left(P_{13} P_{23} R_{23}^{\vee}(y) P_{13} P_{12}\right)\left(P_{12} R_{13}^{\vee}(x y) P_{12}\right) R_{12}^{\vee}(x) \\
& =P_{13} R_{23}(y) R_{13}(x y) R_{12}(x) .
\end{aligned}
$$

Similarly one shows that

$$
R_{23}^{\vee}(x) R_{12}^{\vee}(x y) R_{23}^{\vee}(y)=P_{13} R_{12}(x) R_{13}(x y) R_{23}(y) .
$$

Hence we have

$$
R_{12}^{\vee}(y) R_{23}^{\vee}(x y) R_{12}^{\vee}(x)=R_{23}^{\vee}(x) R_{12}^{\vee}(x y) R_{23}^{\vee}(y) .
$$

We will need the matrices in the second form. For convenience, we drop the ${ }^{\vee}$ symbol and define matrices $R_{i}(x)$ in $\otimes^{\infty} \operatorname{End}(V)$ by

$$
R_{i}(x)=\cdots 1 \otimes R^{\vee}(x) \otimes 1 \cdots,
$$

where $R^{\vee}(x)$ sits in the tensor product of the $i$ th and $(i+1)$ th copy of $\operatorname{End}(V)$. It follows immediately from (19) and (20) that the map $\sigma_{i} \mapsto R_{i}(0)$ defines a representation of the infinite braid group $B_{\infty}$.

We will extend the string model of braids to $R$ matrices. $R_{i}$ will be represented by the same braid projection as the one for $\sigma_{i}$. Moreover, we will write the variable $x$ over the crossing. Then the monoid generated by the $R$ matrices is a homomorphic image of the monoid generated by these symbols with concatenation as for braids as operation. The QYBE equation translates to the equivalence of the 2 pictures in Figure 5.

This means we are allowed to move the middle string over the crossing of the 2 outer strings if the product of the variables of its crossings with the outer strings equals the variable of the fixed crossing.

We define the matrix $R_{i}^{(f)}(x)$ by the picture used for $\sigma_{i}^{(f)}$ where all the crossings have symbol $x$. An algebraic expression for this would be

$$
\begin{aligned}
R_{i}^{(f)}(x)= & \left(R_{i f} R_{i f+1} \cdots R_{(i+1) f-1}\right)\left(R_{i f-1} R_{i f} \cdots R_{(i+1) f-2}\right) \\
& \cdots\left(R_{(i-1) f+1} R_{(i-1) f-2} \cdots R_{i f}\right)
\end{aligned}
$$



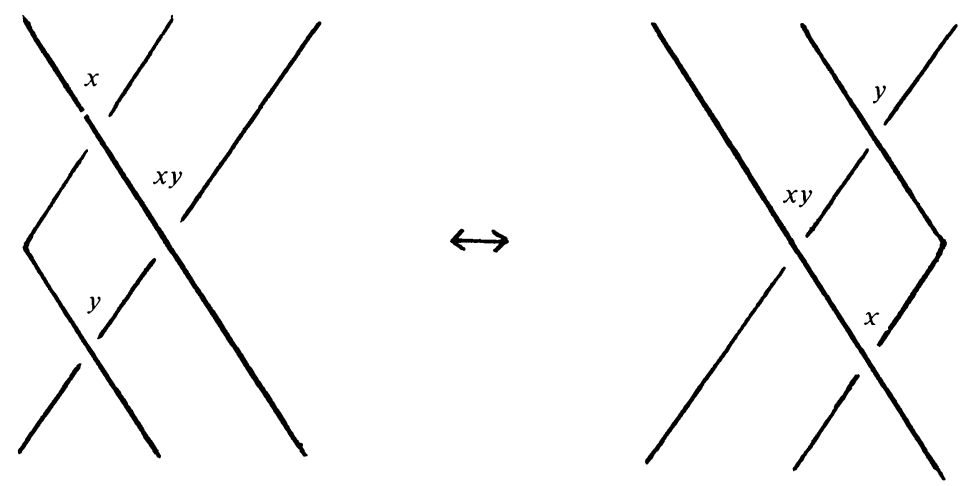

FIGURE 5

As for braids, we can avoid an algebraic proof to show that the newly defined matrices satisfy the QYBE namely that

$$
R_{i}^{(f)}(x) R_{i+1}^{(f)}(x y) R_{i}^{(f)}(y)=R_{i+1}^{(f)}(y) R_{i}^{(f)}(x y) R_{i+1}^{(f)}(x) .
$$

Indeed, to show that the 2 pictures in Figure 6 are equivalent (for the case $f=2$ ) we only have to move the $f$ strings in the middle to the right. As these $f$ strings are parallel, it is easy to see that this can be done by only moving them over $x y$ crossings i.e. by only using moves as described in Figure 5. We obtain

Proposition 11. Let $V$ be a $k$ dimensional vector space and let $R \in \mathrm{Gl}(V \otimes V)$ be a solution of the $Q Y B E$. Then the matrix $R^{(f)} \in$ $\mathrm{Gl}\left(V^{\otimes f} \otimes V^{\otimes f}\right)$ as defined in (21) is a solution of the $Q Y B E$.

It is to be expected that these solutions can be decomposed as it is the case with braids. It is not clear, however, how one can obtain
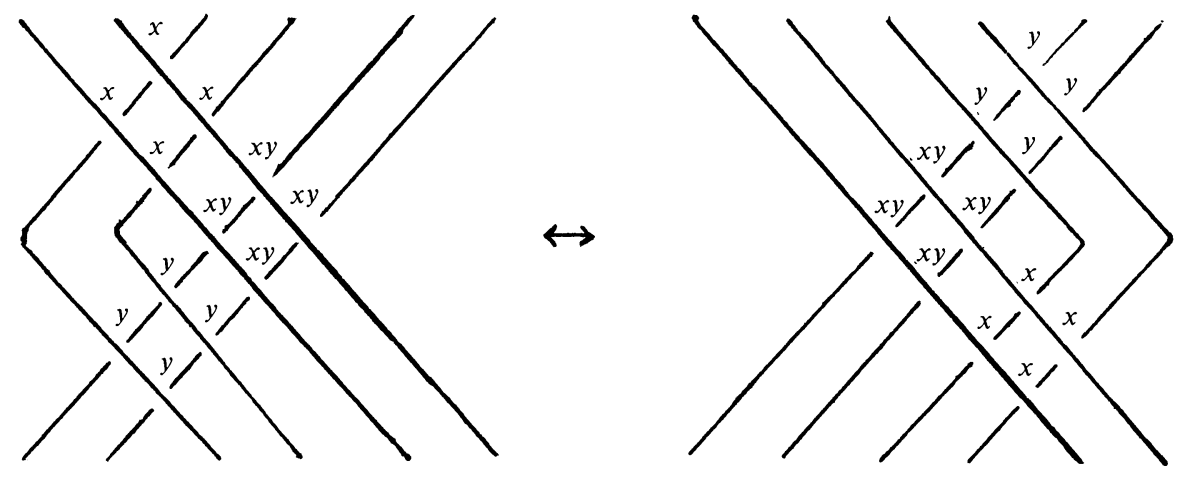

Figure 6 
from this abstractly defined decomposition explicit $R$ matrices by that method. General methods for finding solutions of the QYBE can be found in [D] and [Ji].

It has already been observed by M. Jimbo that his solutions for type $A$ at $x=0$ yield representations of the braid groups into the Hecke algebras of type $A$. The same representations were already obtained earlier by Temperley and Lieb for type $A_{1}$. They were later rediscovered by Pimsner and Popa [PP]. Knowing these representations, it is possible to generalize them to general $A_{n}$ which was also observed by Pimsner and Popa.

If $\left\{f_{i j}, 1 \leq i, j \leq k\right\}$ is a set of matrix units, we can describe these braid representations by the matrix

$$
\begin{aligned}
R=R^{\vee}(0)= & \sum_{i=1}^{k} q f_{i i} \otimes f_{i i} \\
& +\sum_{i<j}(q-1) f_{i i} \otimes f_{j j}-q^{1 / 2}\left(f_{i j} \otimes f_{j i}+f_{j i} \otimes f_{i j}\right) .
\end{aligned}
$$

Note that if $q=1$, the matrix $R$ just becomes the 'flip', i.e. $R(v \otimes w)=w \otimes v$ for $v, w \in V$. Let for the above matrix $R$ the matrices $R_{i}$ be defined as in (20). Then the map $g_{i} \mapsto R_{i}$ extends to a representation $\Phi$ of $H_{\infty}(q)$ on $\bigoplus^{\infty} V$. The projection $P$ onto the eigenspace of $R$ belonging to the eigenvalue -1 is given by

$$
P=\frac{1}{1+q} \sum_{1 \leq i<j \leq k} f_{i i} \otimes f_{j j}+q f_{j j} \otimes f_{i i}+q^{1 / 2}\left(f_{i j} \otimes f_{j i}+f_{j i} \otimes f_{i j}\right) .
$$

Let $M_{k}$ be the set of all $k \times k$ matrices and let $\operatorname{Tr}$ be the usual trace on $M_{k}$ i.e. the sum of their diagonal elements. Let $D$ be the diagonal matrix

$$
D=\sum_{i=1}^{k} q^{i} /\left(q+q^{2}+\cdots+q^{k}\right) f_{i i}
$$

We define the functional $\psi$ on $\otimes_{j \in \mathbf{N}} M_{k}$ by

$$
\psi\left(\bigotimes_{j \in \mathbf{N}} a_{j}\right)=\prod_{j \in \mathbf{N}} \operatorname{Tr}\left(D a_{j}\right)
$$

A straightforward computation shows that

$$
\psi(P)=\left(q-q^{k}\right) /(1+q)\left(1-q^{k}\right)=a_{-k}(q)
$$

and

$$
\psi\left(\left(f_{i j} \otimes 1\right) P\right)=\phi\left(f_{i j}\right) \psi(P) \quad \text { for } 1 \leq i, j \leq k
$$


Let $C_{n}$ be the algebra generated by $R_{1}, R_{2}, \ldots, R_{n-1}$. We can now characterize these representations of braid groups into $C_{n}$ (see also $[\mathbf{A W}],[\mathbf{P P}]$, or $[\mathbf{P}])$.

Proposition 12. The restriction of $\psi$ to $C_{n}$ coincides with the Markov trace on $H_{n}(q)$ under $\Phi$ with

$$
\eta=a_{-k}(q)=\left(q-q^{k}\right) /(1+q)\left(1-q^{k}\right) .
$$

In particular, if $q$ is not an lth root of unity with $l=2,3, \ldots, n$ we have

$$
C_{n} \cong \bigoplus_{\lambda \in \Lambda_{n}, \lambda_{k+1}=0} \pi_{\lambda}\left(H_{n}(q)\right) .
$$

If $q$ is a primitive lth root of unity, let $I_{n}=\left\{a \in C_{n}(q), \psi(a b)=0\right.$ for all $\left.b \in C_{n}\right\}$. Then

$$
C_{n}(q) / I_{q} \cong \bigoplus_{\lambda \in \Lambda_{n}^{(k, l)}} \pi_{\lambda}^{(k, l)}\left(H_{n}(q)\right) .
$$

Proof. Let $a \in\left(\otimes_{j=1}^{n-1} M_{k}\right) \otimes 1$. Then $\psi\left(P_{i} a\right)=\psi(P) \psi(a)$ by (23) and linearity. Hence $\psi$ has to coincide with $\operatorname{tr}_{a_{-k}(q), q}$ by (22) and [W-1], $\S 3$. The statement for $q$ a primitive root of unity follows from [W-1], Theorem 3.6 (the proof there for $q=e^{2 \pi i / l}$ goes through for any arbitrary primitive $l$ th root of unity with the exception of the positivity and unitarity statements).

If $q=1$, the matrices $R_{i}$ just permute the factors in $V^{\otimes n}$. So the statement follows from Schur-Weyl duality. To show the general statement, let $z_{\lambda}$ be the central idempotent of $H_{n}$ belonging to the Young diagram $\lambda$. If $H_{n}(q)$ is semisimple, $z_{\lambda}(q)$ is well defined and so is $\Phi\left(z_{\lambda}(q)\right)$. Let $\operatorname{Tr}$ be the usual nonnormalized trace on $M_{k}^{\otimes n}=M_{n k}$. Observe that $\operatorname{Tr}\left(\Phi\left(z_{\lambda}(q)\right)\right)$ is the rank of $\Phi\left(z_{\lambda}(q)\right)$ on $V^{\otimes n}$ and that $q \mapsto \operatorname{Tr}\left(\Phi\left(z_{\lambda}(q)\right)\right)$ is continuous whenever $z_{\lambda}(q)$ is well defined. Hence in this case the rank of $z_{\lambda}(q)$ equals the one of $z_{\lambda}(1)$. Counting dimensions, it follows from the case $q=1$ that

$$
\sum_{\lambda \in \Lambda_{n}, \lambda_{k+1}=0} \Phi\left(z_{\lambda}(q)\right)=\left.1\right|_{V^{\otimes n}}
$$

and that $\Phi\left(z_{\lambda}(q)\right) \neq 0$ for all the Young diagrams appearing in the sum above.

By Schur-Weyl duality, any minimal idempotent $p$ in $\Phi\left(H_{f}(1)\right)$ can be interpreted as a projection onto an $\rho^{\otimes f}(\mathrm{SU}(k))$-invariant subspace of $V^{\otimes f}$. The corresponding irreducible representation of $\mathrm{SU}(k)$ 
is labelled by the Young diagram belonging to $p$. So it follows from Propositions 2 and 4 and Theorem 8.

COROLLARY. There exists for each irreducible representation $\pi^{(\lambda)}$ of $\mathrm{SU}(k)$, labelled by the Young diagram $\lambda$, a one variable link invariant. It can be either obtained from solutions of the QYBE corresponding to this representation or from the link invariant $L^{(\lambda)}$ of Theorem 8 with $\eta=a_{-k}(q)$.

In the special case $\mathrm{SU}(2)$ these link invariants were also obtained independently by Jones who proceeded directly from Jimbo's explicit solutions of the QYBE for higher representations of $\mathrm{SU}(2)$. Additional information of braid representations coming from solutions of QYBE is used in [J-2] to construct state models for link invariants. Similar models have been defined subsequently by Turaev in [T] using solutions of the QYBE for other Lie types. The corresponding algebras will be studied in detail in [W-3]. More recently, link invariants have been obtained for higher representations of Lie algebras directly from the quantum group approach by Reshetikhin [Re] and Rosso [Ro].

\section{REFERENCES}

[AW] Y. Akutsu and M. Wadati, Exactly solvable models and new link polynomials, J. Phys. Soc. of Japan, 56 No. 9, (1987), 3039-3051.

[B] J. Birman, Braids, links and mapping class groups, Ann. Math. Studies 82, Princeton Univ. Press, (1974).

[BW] J. Birman and H. Wenzl, Braids, link polynomials, and a new algebra, Trans. Amer. Math. Soc., 313 (1989), 249-273.

[Bk] N. Bourbaki, Algebre, I-II.

[BZ] G. Burde and H. Zieschang, Knots, de Gruyter (1985).

[D] V. Drinfeld, Quantum groups, Proceedings of the ICM, Berkeley 1986, 798-820.

[FYHLMO] P. Freyd, D. Yetter, J. Hoste, W. B. R. Lickorish, K. Millett and A. Ocneanu, A new polynomial invariant of knots and links, Bull. Amer. Math. Soc., 12, No. 2, (1985), 239-246.

[G] A. Gyoja, $A$ q-analogue of Young symmetrizer, Osaka J. Math., 23 (1986), 841-852.

[H] P. N. Hoefsmit, Representations of Hecke algebras of finite groups with $B N$ pairs of classical type, thesis, University of British Columbia, (1974).

[Ji-1] M. Jimbo, Quantum R-matrix for the generalized Toda system, Comm. Math. Phys., 102, 4 (1986), 537-547.

[Ji-2] $\quad$ A $q$ analogue of $U(\mathrm{gl}(N+1))$, Hecke algebras and the YangBaxter equation, Lett. Math. Phys., 10 (1985), 63-69.

[J-1] V. F. R. Jones, Hecke algebra representations of braid groups and link polynomials, Ann. Math., 126 (1987), 335-388. 

, On knot invariants related to some statistical mechanics models, Pacific J. Math., 137 (1989), 311-334.

[K-1] L. H. Kauffman, State models and the Jones polynomial, Topology, 26 No. 3 (1987), 395-407.

[K-2] _ An invariant of regular isotopy, Trans. Amer. Math. Soc., 318 (1990), 417-471.

[KS] P. P. Kulish and E. P. Sklyanin, On the solutions of the Yang-Baxter equation, J. Soviet Math., 19 (1982).

[Mc] I. MacDonald, Symmetric Functions and Hall Polynomials, Clarendon Press; Oxford, 1979.

[M] G. Mackey, The theory of unitary group representations, Chicago Lectures in Mathematics (1976).

[MS] H. R. Morton and H. B. Short, The 2-variable polynomial of cable knots, Math. Proc. Camb. Phil. Soc., (to appear).

[M] J. Murakami, The parallel version of link invariants, preprint.

[P] V. Pasquier, Continuum limit of lattice models built on quantum groups, SACLAY preprint 87-125.

[PP] M. Pimsner and S. Popa, Entropy and index for subfactors, Ann. scient. Ec. Norm. Sup., $4^{e}$ serie t. 19 (1986), 57-106.

[Re] N. Yu. Reshetikhin, Quantized universal enveloping algebras, the YangBaxter equation and invariants of links, LOMI preprint.

[R.] G. de Robinson, Representation theory of the symmetric group, University of Toronto Press (1961).

[Ro] M. Rosso, Groupes quantiques et modeles a vertex de V. Jones en théorie des noeuds, C. R. Acad. Sci. Paris, 307 (1988), 207-210.

[T] V. G. Turaev, The Yang-Baxter equation and invariants of links, Invent. Math., 92 (1988), 527-553.

[W-1] H. Wenzl, Hecke algebras of type $A_{n}$ and subfactors, Invent. Math., 92 (1988), 349-383.

[W-2] _ On the structure of Brauer's centralizer algebras, Ann. of Math., 128 (1988), 173-193.

[W-3] __ Subfactors and quantum groups for Lie type $B, C$ and $D$, preprint.

[W-4] _ Derived link invariants and subfactors, Conference proceedings of UK-US Joint Seminar in Operator Algebras 1987, LMS Lecture Notes Nr. 135/136, Cambridge Press, (to appear).

Received November 15, 1988. Supported in part by NSF grant \#DMS88-05378.

University of California, SAN Diego

LA Jolla, CA 92093 


\section{PACIFIC JOURNAL OF MATHEMATICS EDITORS}

\author{
V. S. VARAdarajaN \\ (Managing Editor) \\ University of California \\ Los Angeles, CA 90024-1555-05 \\ Herbert Clemens \\ University of Utah \\ Salt Lake City, UT 84112 \\ Thomas ENRIGHT \\ University of California, San Diego \\ La Jolla, CA 92093
}

R. FINN

Stanford University

Stanford, CA 94305

HermanN FlaschKa

University of Arizona

Tucson, AZ 85721

VAUGHan F. R. Jones

University of California

Berkeley, CA 94720

STEVEN KeRCKHOFF

Stanford University

Stanford, CA 94305
C. C. Moore University of California Berkeley, CA 94720

MARTIN SCHARLEMANN University of California Santa Barbara, CA 93106

\section{HAROLD STARK}

University of California, San Diego La Jolla, CA 92093

\section{ASSOCIATE EDITORS}
R. ARENS
E. F. BECKeNBACH
B. H. NeumanN
F. WolF
(1904-1989)
K. Yoshida (1906-1982)

\section{SUPPORTING INSTITUTIONS}

UNIVERSITY OF ARIZONA

UNIVERSITY OF BRITISH COLUMBIA

CALIFORNIA INSTITUTE OF TECHNOLOGY

UNIVERSITY OF CALIFORNIA

MONTANA STATE UNIVERSITY

UNIVERSITY OF NEVADA, RENO

NEW MEXICO STATE UNIVERSITY

OREGON STATE UNIVERSITY
UNIVERSITY OF OREGON

UNIVERSITY OF SOUTHERN CALIFORNIA

STANFORD UNIVERSITY

UNIVERSITY OF HAWAII

UNIVERSITY OF TOKYO

UNIVERSITY OF UTAH

WASHINGTON STATE UNIVERSITY

UNIVERSITY OF WASHINGTON 


\section{Pacific Journal of Mathematics}

Vol. 145, No. 1 September, 1990

Sheldon Jay Axler and Allen Lowell Shields, Extensions of harmonic and

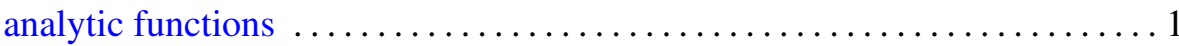

Labib Haddad and Yves Sureau, Les cogroupes et la construction de

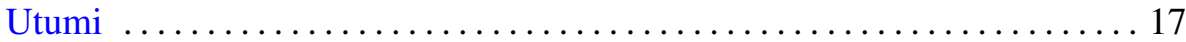

John Hutchinson, Poincaré-Sobolev and related inequalities for submanifolds of $\mathbf{R}^{N}$

Yuk Jaum Leung and Glenn E. Schober, Some coefficient problems and

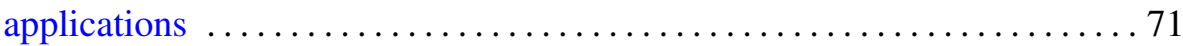

Daniel Ruberman, Seifert surfaces of knots in $S^{4} \ldots \ldots \ldots \ldots \ldots \ldots \ldots \ldots$

Joel Harold Shapiro and Carl Sundberg, Isolation amongst the

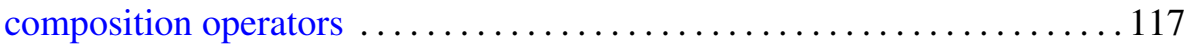

Hans Wenzl, Representations of braid groups and the quantum Yang-Baxter

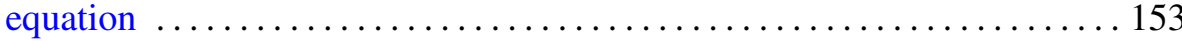

Shuang Zhang, Diagonalizing projections in multiplier algebras and in matrices over a $C^{*}$-algebra 